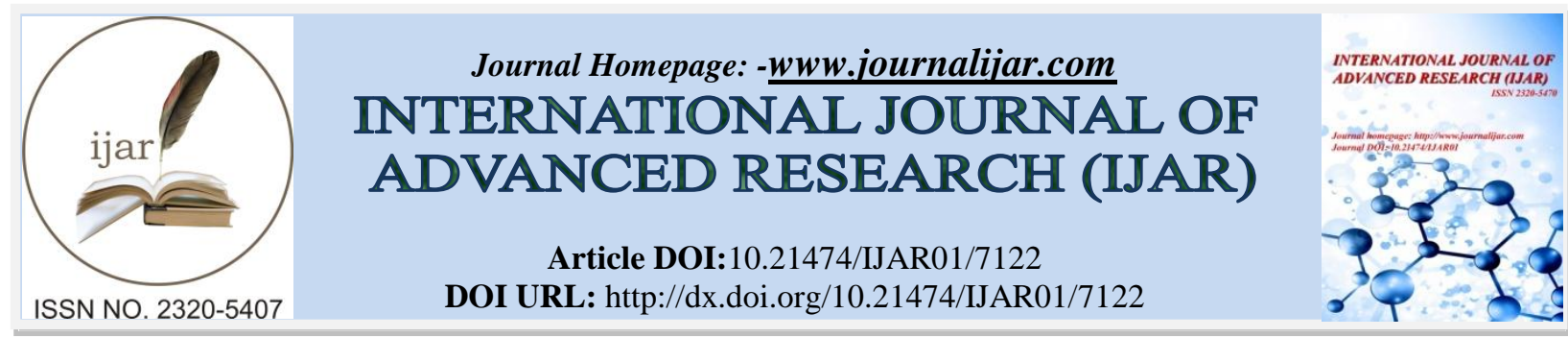

RESEARCH ARTICLE

\title{
THE EFFECT OF A NEW PROBIOTIC PRODUCT LINE IN PATIENTS WITH IRRITABLE BOWEL SYNDROME: CLINICAL OBSERVATION.
}

VentsislavNakov and RadislavNakov.

Clinic of Gastroenterology; “TsaritsaYoanna -ISUL" University Hospital, Medical University of Sofia, Bulgaria.

\section{Manuscript Info}

Manuscript History

Received: 17 March 2018

Final Accepted: 19 April 2018

Published: May 2018

\section{Abstract}

\section{Background}

It has been proven for many years that probiotics play pivotal role in the treatment of IBS patients. However it is still not very clear which strains are more beneficial than others in the treatment of irritable bowel syndrome (IBS).Our aim in the current clinical observation was to follow up the effect of a novel probiotic product line available on the Bulgarian market - Biopron IB-Symbio (Walmark, Czech Republic) in the treatment of IBS patients.

\section{Methods}

This prospective clinical observation included 45 consecutive patients divided into three groups: group $1-15$ patients with diarrhea predominant post-infectious IBS (IBS-D), group 2 - 15 with constipation-predominant IBS (IBS-C) and group 3 - 15 IBS patients meeting Rome III but not meeting Rome IV criteria. Four week monotherapy with a new probiotic line was commenced in every group. The symptoms were evaluated before and after treatment. Moreover in IBS-D group fecal calprotectin (FCP) was monitored

\section{Results}

Significant reduction of FCP mean levels was observed in group 1 from $152 \mu \mathrm{g} / \mathrm{g}$ at week 0 to $64 \mu \mathrm{g} / \mathrm{gat}$ week 4 (p<0,001). After probiotic treatment diarrhea was present only in $3(20 \%)$ patients, and pain in 5 (33\%) of the patients. In group 2 constipation was present after treatment just in $2(13 \%)$ patients and abdominal pain in $4(27 \%)$ of them. In group 3 - at 4 week after treatment discomfort was present in 3 $(20 \%)$ patients, bloating in $2(13 \%)$ patients and change in frequency of stool in $4(27 \%)$

\section{Conclusion}

The current clinical observation shows that Biopron probiotic product line significantly improves the symptoms in IBS patients. Moreover, it reduces the inflammatory activity in patients with post-infectious IBS.Additional multicenter observations with larger number of patients from different regions are needed to obtain even more accurate data 
Irritable bowel syndrome (IBS) is a common functional condition of unclear, complex pathophysiology that has a large impact on quality of life and both direct and indirect healthcare costs $(1,2)$. It is the most frequent diagnosed gastrointestinal disorder and one of the most common functional disorders of the gastrointestinal tract (GIT) in the clinical practice ( $41 \%$ from all patients with GIT disorders). The prevalence of IBS in the developed countries is between $5 \%$ and $20 \%$. (3).

IBS demonstrates variable clinical course with a broad spectrum of gastrointestinal and extraintestinal symptoms. It is a chronic disease but with a good prognosis and does not lead to increased mortality compared to general population (4).

Recently, there are growing evidences about the role of acute gastrointestinal infections in the development of IBS post-infectious IBS. It is observed in $10-30 \%$ of the patients following acute episode of infectious gastroenteritis. The percentage in Bulgaria is even higher $-44 \%$ of patients with post-infectious enteritis (5). The underlying causes consist of a variety of chronic and persistent clinical manifestations of the intestinal inflammation after acute infection; morphological lesions and disintegration of the intestinal mucosa; impairment of barrier function with increased intestinal permeability and excessive lumen exposure to antigens; the entry of these antigens into the lining and activation of the local immune, endocrine and autoimmune system; secretion of proinflammatory factors, gastrointestinal hormones, physiologically active substances, etc. (5).

In 2016 the "Rome foundation" accepted the new criteria for IBS diagnostics - Rome IV (Table 1) (3). The main difference with the previous version of Rome criteria from 2006 (Rome III (6))(Table 2) is that the symptom discomfort is eliminated, and it is already assumed that a patient has IBS only if recurrent abdominal pain is present at least once in a week instead of at least three times per month.

A potential problem arises from this last revision due to the existence of a group of patients that demonstrate symptoms correlating to the Rome III criteria but still do not fulfill the Rome IV criteria. (7).

Table 1:-Rome IV (2016) diagnostic criteria for Irritable Bowel Syndrome

Recurrent abdominal pain at least once in a week for the last 3 months related with one of the following:

1. Defecation

2. Change of the frequency of defecation

3. Change of the consistency (type) of the stools

All criteria should be fulfilled for minimum 3 months prior to the visit of physician`s office with symptoms history of at least 6 months.

Table 2:-Rome III (2006) diagnostic criteria for Irritable Bowel Syndrome

Recurrent abdominal pain or discomfort $\dagger$ more than 3 days per month over the previous 3 months associated with two or more of the following:

1. Improvement with defecation

2. Onset associated with a change in frequency of stool

3. Onset associated with a change in form or appearance of stool

Symptom onset greater than 6 months prior to the diagnosis, with the above criteria fulfilled for the past 3 months

$\dagger$ Discomfort means an uncomfortable sensation not described as pain

There are many pharmacological and nonpharmacological treatment options for IBS, each of which demonstrate different outcomes (8). Due to the heterogeneity of symptoms IBS should be treated individually in each patient. Of key importance is the doctor-patient communication together with proper training and recommendations about suitable diet and physical exercises. 
It has been proven for many years that probiotics play pivotal role in the treatment of IBS patients. However it is still not very clear which strains are more beneficial than others in the treatment of IBS (9). In the clinical practice symbiotics, containing probiotics together with prebiotics are commonly prescribed.

Our aim in the current clinical observation was to follow up the effect of a novel probiotic product line available on the Bulgarian market - Biopron IB-Symbio (Walmark, Czech Republic) in the treatment of IBS patients.

\section{Materials and methods:-}

This prospective clinical observation included 45 consecutive patients who visited the Clinic of Gastroenterology at "TsaritsaYoanna -ISUL" University Hospital between December 2017 and March 2018. The patients were divided into three groups.

Group 1 - 15 patients with post-infectious diarrhea-predominant IBS(IBS-D) - 9 women, at mean age of 36 (18-56 years) were assigned to monotherapy with Biopron S. boulardii 2 capsules per day for 4 weeks. Biopron S. boulardii is a symbiotic that contains fructooligosaccharides as prebiotic and the following probiotic strains (10x10 9 CFUs) Saccharomyces boulardii, Lactobacillus acidophilus and Lactobacillus rhamnosus (Table 3).

Table 3:-Ingredients of Biopron Saccharomyces boulardii

\begin{tabular}{|l|l|}
\hline Daily dosage of 2 capsules contains: & Quantity of 2 capsules \\
\hline $\begin{array}{l}\text { Saccharomyces boulardii } \\
\text { Lactobacillus acidophilus } \\
\text { Lactobacillus rhamnosus }\end{array}$ & $10 \times 10^{9} \mathrm{CFU}$ \\
\hline Fructooligosaccharides & \\
\hline$* \mathrm{CFU}$ - colony forming units & $238,6 \mathrm{mg}$ \\
\hline
\end{tabular}

IBS diagnosis was made by Rome IV criteria (Table 1). Inclusion criteria were normal ileocolonoscopy, normal results from laboratory blood tests, normal microbiology cultures and values of fecal calprotectin (FCP) above 70 $\mu \mathrm{g} / \mathrm{g}$ as an evidence for low-grade intestinal inflammation. FCP levels were measured by quantitative immunochromatographic test Quantum Blue (Bühlmann Laboratories AG, Switzerland). We followed all the instructions provided by the manufacturer (10). The exclusion criteria were use of NSAIDs, proton pump inhibitors (PPI) and antibiotics within 4 weeks before the observation.

Group 2:-15 patients with constipation-predominant IBS BS-C) - 10 women, at mean age of 39 years (20-58 years) were assigned to monotherapy with Biopron Fibers 2 sachets daily for 4 weeks. Biopron Fibers contains soluble fibers (psyllium), inulin as prebiotic and 9 strains probiotics $\left(4 \times 10^{9}\right.$ CFUs) - Bifidobacteriumbifidum, Bifidobacteriumbreve, Bifidobacteriumlongum, Lactobacillus acidophilus, Lactobacillus casei, Lactobacillus plantarum, Lactobacillus rhamnosus, Lactococcuslactis ssp. Lactis, Streptococcus thermophilus (Table 4).

Table 4:-Ingredients of BiopronFibres

\begin{tabular}{|l|l|}
\hline Ingredients: & Quantity of 1 sachet \\
\hline Bifidobacteriumbifidum & $4 \times 10^{9} \mathrm{CFU}$ \\
Bifidobacteriumbreve & \\
Bifidobacteriumlongum & \\
Lactobacillus acidophilus & \\
Lactobacillus casei & \\
Lactobacillus plantarum & \\
Lactobacillus rhamnosus & \\
Lactococcuslactis ssp. Lactis & \\
Streptococcus thermophilus & \\
\hline Psyllium husk & $3475,0 \mathrm{mg}$ \\
\hline Inulin & $1500,0 \mathrm{mg}$ \\
\hline$*$ CFU - colony forming units & \\
\hline
\end{tabular}


The diagnosis IBS-C was made just by Rome IV (Table 1). Inclusion criteria were normal ileocolonoscopy, normal results from laboratory blood tests and normal microbiology cultures. As constipation one defecation in 3 or more days was accepted.

Group 3:-15 patients (10 women, mean age 34 years (19 - 46 years)) with IBS that meet Rome III criteria but do not meet Rome IV criteria were treated with monotherapy of Biopron Enzymes 2 capsules daily for 4 weeks. Biopron Enzymes contains fructooligosaccharides as prebiotic, digestive enzymes (amylase, lipase, protease) and 9 strains of healthy micoorganisms (10x10 ${ }^{9}$ CFUs) - Bifidobacteriumbifidum, Bifidobacteriumbreve, Bifidobacteriumlongum, Lactobacillus acidophilus, Lactobacillus casei, Lactobacillus plantarum, Lactobacillus rhamnosus, Lactococcuslactis ssp. Lactis, Streptococcus thermophilus (Table 5).

Table 5:-Ingredients of Biopron Enzymes

\begin{tabular}{|l|l|}
\hline Daily dosage of 2 capsules contains: & Quantity of 2 capsules \\
\hline Bifidobacteriumbifidum & $9 \times 10^{9} \mathrm{CFU}^{*}$ \\
Bifidobacteriumbreve & \\
Bifidobacteriumlongum & \\
Lactobacillus acidophilus & \\
Lactobacillus casei & \\
Lactobacillus plantarum & \\
Lactobacillus rhamnosus & \\
Lactococcuslactis ssp. Lactis & \\
Streptococcus thermophilus & \\
\hline $\begin{array}{l}\text { Digestive enzymes } \\
\text { Amylase }\end{array}$ & $55,8 \mathrm{mg}$ \\
Lipase & \\
Protease & \\
\hline Fructooligosaccharides & $259,2 \mathrm{mg}$ \\
\hline$*$ CFU - colony forming units & \\
\hline
\end{tabular}

The diagnosis was based on Rome III criteria (Table 2), normal ileocolonoscopy, normal laboratory and microbiology results.

\section{Results:-}

All patients from group 1 (IBS-D) demonstrated diarrhea and abdominal pain before the commencement of monotherapy with Biopron S. boulardii. Four weeks later diarrhea was present only in $3(20 \%)$ patients, and pain in $5(33 \%)$ patients (Table 6$)$.

Table 6:-Presence of diarrhea and abdominal pain in patients with IBS-D before and after 4 week treatment with Biopron S. boulardii

\begin{tabular}{|l|l|l|l|l|}
\hline \multirow{2}{*}{ Symptoms } & \multicolumn{2}{|c|}{ Before treatment } & \multicolumn{2}{c|}{ After treatment } \\
\cline { 2 - 5 } & $\mathbf{n}$ & $\mathbf{\%}$ & $\mathbf{n}$ & $\mathbf{2 0}$ \\
\hline Diarrhea & $\mathbf{1 5}$ & $\mathbf{1 0 0}$ & $\mathbf{3}$ & $\mathbf{3 3}$ \\
\hline Pain & $\mathbf{1 5}$ & $\mathbf{1 0 0}$ & $\mathbf{5}$ & \\
\hline
\end{tabular}

Significant reduction of FCP mean levels was observed - from $152 \mu \mathrm{g} / \mathrm{g}$ at week 0 to $64 \mu \mathrm{g} / \mathrm{g}$ at week 4 (p<0,001). (Figure 1). 


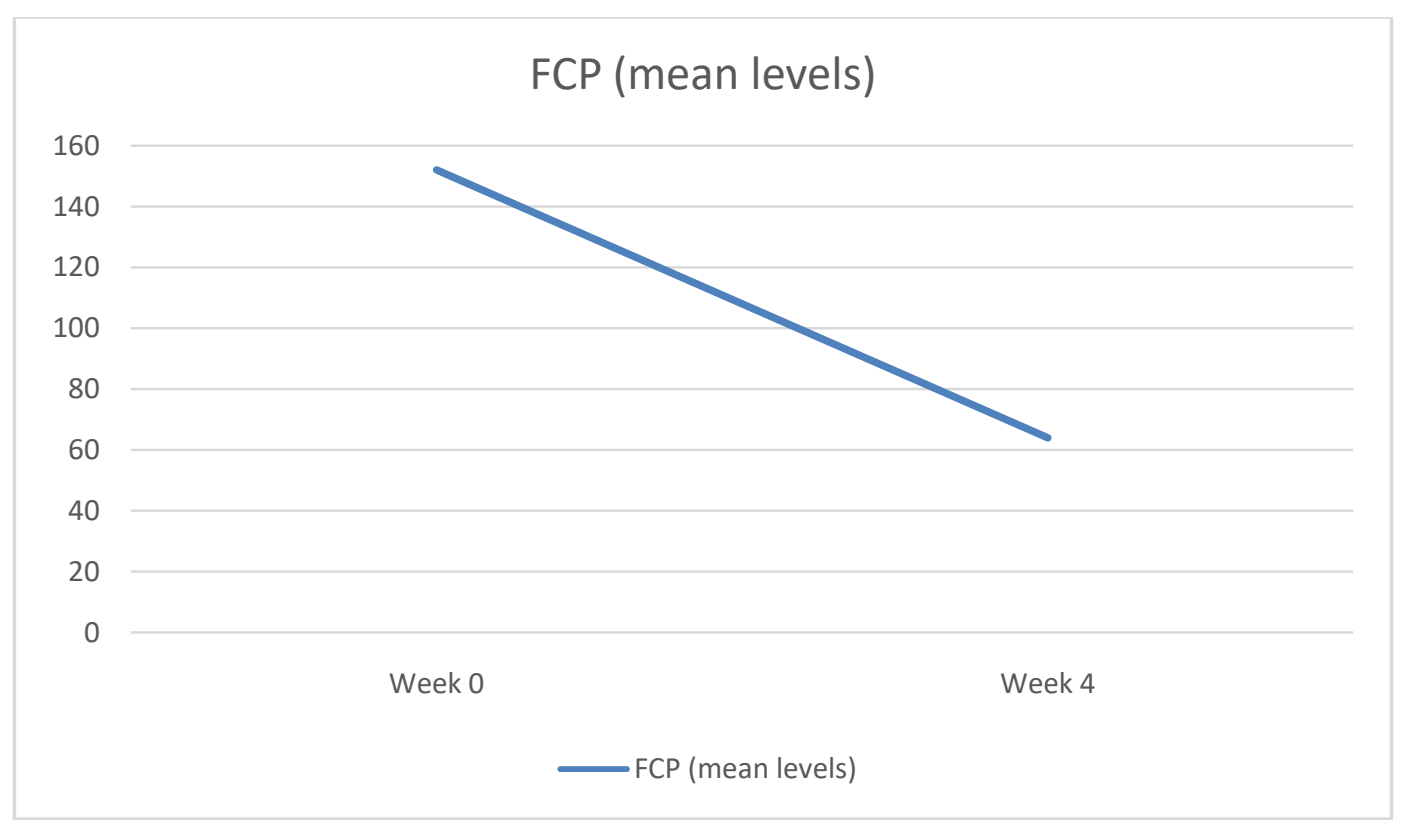

Figure 1:-Mean levels of fecal calprotectin in patients with IBS-D before and after treatment with Biopron S. boulardii

All patients in group 2 (IBS-C) had constipation and abdominal pain in the beginning of the clinical observation. After 4 weeks of monotherapy with Biopron Fibers constipation was still present in 2 (13\%) patients and abdominal pain in just $4(27 \%)$ of them (Table 7$)$.

Table 7:-Presence of constipation and abdominal pain in patients with IBS-C before and after 4 week treatment with Biopron Fibers

\begin{tabular}{|l|l|l|l|l|}
\hline \multirow{2}{*}{ Symptoms } & Before treatment & After treatment \\
\cline { 2 - 5 } & n & $\%$ & n & $\%$ \\
\hline constipation & 15 & 100 & 2 & 13 \\
\hline pain & 15 & 100 & 4 & 27 \\
\hline
\end{tabular}

All patients in group 3 had abdominal discomfort, bloating and change in frequency of stool before treatment with Biopron Enzymes. At week 4 after treatment discomfort was still present in $3(20 \%)$ patients, bloating in $2(13 \%)$ patients and change in frequency of stool in 4 (27\%) (Table 8).

Table 8:-Presence of abdominal discomfort, bloating and change in frequency of stool in patients with IBS fulfilling Rome III criteria before and after 4 week treatment with Biopron Enzymes

\begin{tabular}{|l|l|l|l|l|}
\hline \multirow{2}{*}{ Symptoms } & \multicolumn{2}{|l|}{ Before treatment } & After treatment \\
\cline { 2 - 6 } & $\mathbf{n}$ & $\%$ & $\mathbf{n}$ & $\%$ \\
\hline Discomfort & 15 & $\mathbf{1 0 0}$ & $\mathbf{3}$ & $\mathbf{2 0}$ \\
\hline Bloating & 15 & $\mathbf{1 0 0}$ & $\mathbf{2}$ & $\mathbf{1 3}$ \\
\hline Change in frequency of stool & 15 & $\mathbf{1 0 0}$ & $\mathbf{2}$ & 27 \\
\hline
\end{tabular}

\section{Discussion:-}

The effect of symbiotics in IBS patients is well studied (9). Probiotics are capable of inhibiting the adhesion and the invasion of the pathogens to the epithelial wall, to eradicate microbes via production of antimicrobial products, to stimulate the cell turnover and the immune response, to induce the production of anti-inflammatory cytokins (IL10, TGF, IgA, $\downarrow$ TNF) and to stimulate the phagocytic function of the neutrophils (11). Furthermore, the consumption of 
trans-galactooligosaccharides stimulates specific intestinal Bifidobacteria in patients with IBS and effectively alleviates the symptoms of the diseases $(12,13)$.

The current clinical observation demonstrates that the intake of Biopron S. boulardii for 4 weeks by patients with post-infectious IBS leads to improvement of diarrhea in $80 \%$ of the patients, alleviation of abdominal pain in $67 \%$ of the patients and reduction of mean FCP levels therefore the level of intestinal inflammation.

In our previous studies we demonstrated that probiotics lead to a reduction in inflammatory activity in patients with post-infectious IBS, resulting in a reduction in FCP values and reduction or even disappearance of clinical symptoms (11). A number of other studies have also shown that the intake of symbiotics in IBS patients leads to improvement of symptoms and quality of life $(9,14)$.

In patients with IBS-C 4-week therapy with Biopron Fibers leads to reduction of constipation in $87 \%$ of the patients and alleviation of pain in $73 \%$ of the patients. Having in mind that in clinical practice, gastroenterologists are not prescribing symbiotics very often to patients with chronic constipation, we find these results quite important. The study outcomes clearly demonstrated that the combination between psyllium and symbiotic improves the symptoms in patients with IBS-C.

The effects of psyllium in chronic constipation has been known for decades (15). A number of studies have demonstrated that psyllium has the ability to influence abdominal pain and discomfort as well as the difficulty of defecation (16). Colecchia et al. found that symbiotic containing Bifidobacteriumlongum (also present in Biopron Fibers) and short-chained oligosaccharide prebiotic leads to increase in the frequency of defecation in IBS-C patients, as well as reduction in abdominal pain and bloating (17).

With the introduction of the new Rome IV diagnostic criteria several important for the clinical practice questions arose. It was observed that due to the stringency of the new criteria the frequency of IBS dropped significantly. Palson et al. demonstrated that according to the Rome III criteria $10,7 \%$ of the population of Canada, USA and Great Britain was diagnosed with IBS. Meanwhile this number was 5,7\% following the Rome IV criteria (18). This study clearly demonstrates that there is a significant group of patients meeting Rome III criteria but not meeting Rome IV. According to a recent study by Patcharatrakul et al. (7) $40 \%$ of patients responding to Rome III did not meet Rome IV due to lack of abdominal pain, lower incidence of pain and/or lack of relationship between pain and defecation. Hence, it is quite normal to ask ourselves whether these patients have a disease or their complaints are due to other factors (e.g. diet) and if they have a disease - what is it and how to deal with its symptoms?

Following the above mentioned assumptions we followed-up 15 patients meeting Rome III criteria but not meeting Rome IV and offered them a monotherapy with Biopron Enzymes. After 4 weeks of treatment we found amelioration of abdominal discomfort in $80 \%$ of the patients, reduction of bloating in $87 \%$ and normalization of frequency of stool in $73 \%$ of the patients.

Several other studies also demonstrate a positive effect of digestive enzymes on the symptoms of IBS patients (19, 20). A study among 18 healthy volunteers has shown that digestive enzyme intake along with a high-calories diet leads to significant reductions in bloating, meteorism and abdominal discomfort (19).

\section{Conclusions:-}

The current clinical observation shows that Biopron probiotic product line significantly improves the symptoms in IBS patients. Moreover, Biopron S. Boulardii reduces the inflammatory activity in patients with post-infectious IBS. Additional multicenter observations with a larger number of patients from different regions are needed to obtain even more accurate data on the effect of Biopron in patients with IBS.

\section{References:-}

1. Spinelli A. Irrirablebowel syndrome. Clin Drug Invest 2007: 27 (1): 15-33

2. Camilleri M, Dubois D, Coulie B, et al. Prevalence and socioeconomic impact of upper gastrointestinal disorders in the United States: Results of the US Upper Gastrointestinal Study. ClinGastroenterol. Hepatol. 2005;3:543-552.

3. Drossman DA. The Rome IV Committees, editor. History of functional gastrointestinal symptoms and disorders and chronicle of the Rome Foundation. In: Drossman DA, Chang LC, Kellow WJ, Tack J, Whitehead WE, editors. Rome IV functional gastrointestinal disorders: disorders of gut-brain interaction. I. Raleigh, NC: The Rome Foundation; 2016. pp. 549-576. 
4. Moayyedi P, Mearin F, Azpiroz F, et al. Irritable bowel syndrome diagnosis and management: A simplified algorithm for clinical practice. UEG Journal. 2017; 5(6): 773-788.

5. Nakov VN, Gerova VA, Nakov RV.Fecal Calprotectinis a reliable non-invasive marker for assessment of intestinal inflammation in patients with irritable bowel syndrome. Compt rend AcadBulgSci, 66, 2013, 9, 1339-1344

6. Drossman DA, moderator. AGA Clinical Symposium - Rome III: New Criteria for the Functional GI Disorders. Program and abstracts of Digestive Disease Week; May 20-25, 2006; Los Angeles, California. [Sp461-469]

7. Patcharatrakul T. et al Application of Rome III vs. Rome IV Diagnostic Criteria for Irritable Bowel Syndrome (IBS) in Clinical Practice: is the Newer the Better? Gastroenterology. 152. S717. 10.1016/S0016-5085(17)32494-0.

8. Simrén, M, Törnblom, H, Palsson, OS Management of the multiple symptoms of irritable bowel syndrome. Lancet GastroenterolHepatol 2017; 2: 112-122.

9. Ford, AC, Quigley, EM, Lacy, BE Efficacy of prebiotics, probiotics, and synbiotics in irritable bowel syndrome and chronic idiopathic constipation: Systematic review and meta-analysis. Am J Gastroenterol 2014; 109: 1547-1561.quiz 1546, 1562.

10. Bühlmann Laboratories AG. Quantum Blue Calprotectin: Quantitative lateral flow assay [pamphlet]. Schönenbuch: Bühlmann Laboratories AG; 2009.

11. VelikovaT., NakovV, GeorgievaR, Toumangelova-YuzeirK, Ivanova-TodorovaE, NakovR et al. Immunomodulating properties of a novel synbiotic on healthy persons. Compt rend AcadBulgSci, 68, 2015, 10, 1321-1326.

12. Curro D, Ianiro G, Pecere S, Bibbo S, Cammarota G. Probiotics, fi bre and herbal medicinal products for functional and inflammatory bowel disorders. Br J Pharmacol 2016; doi: 10.1111/bph. 13632

13. Silk DBA, Davis A, Vulevic J, Tzortzis G, Gibson GR. Clinical trial: the effects of a trans-galactooligosaccharide prebiotic on faecalmicrobiota and symptoms in irritable bowel syndrome. Aliment PharmacolTher 2009; 29(5): 50818

14. Guglielmetti S, Mora D, Gschwender M, Popp K. Randomised clinical trial: Bifidobacteriumbifidum MIMBb75 significantly alleviates irritable bowel syndrome and improves quality of life--a double-blind, placebo-controlled study. Aliment PharmacolTher. 2011 May;33(10):1123-32

15. Fenn GC, Wilkinson PD, Lee CE, Akbar FA. A general practice study of the efficacy of Regulan in functional constipation. Br J ClinPract. 1986 May;40(5):192-7.

16. Moayyedi, P, Quigley, EM, Lacy, BE The effect of fiber supplementation on irritable bowel syndrome: A systematic review and meta-analysis. Am J Gastroenterol 2014; 109: 1367-1374

17. Colecchia A, Vestito A, La Rocca A, Pasqui F, Nikiforaki A, Festi D. Effect of a symbiotic preparation on the clinical manifestations of irritable bowel syndrome, constipation-variant. Results of an open, uncontrolled multicenter study. Minerva GastroenterolDietol 2006; 52(4): 349-58

18. Palsson et al. Population prevalence of Rome IV and Rome III Irritable Bowel Syndrome in the US, Canada and UK. DDW 2016. Mon 1642.

19. Suarez F, Levitt MD, Adshead J, Barkin JS. Pancreatic supplements reduce symptomatic response of healthy subjects to a high fat meal. Dig Dis Sci 1999; 44(7):1317-21

20. Leeds JS, Hopper AD, Sidhu R, Simmonette A, Azadbakht N, Hoggard N, Morley S, Sanders DS. Some Patients With Irritable Bowel Syndrome May Have exocrine Pancreatic Insufficiency. ClinGastroenterolHepatol 2010; 8: 433438 